\title{
A scenario for writing creative scenarios
}

\author{
Michael D. Murphy ${ }^{1}$ [D $\cdot$ Don R. Day ${ }^{2}$
}

Received: 26 January 2021 / Accepted: 7 April 2021 / Published online: 7 June 2021

(c) The Author(s), under exclusive licence to Springer Nature Singapore Pte Ltd. 2021

\begin{abstract}
This paper examines the elements of a pathway to writing the bold, innovative scenarios necessary to promote sustainable socio-ecological integration. Innovative scenarios incorporate three virtues essential to making knowledge systemically useful: creativity, collaboration, and communication. The main features of such a scenario-writing process include: (1) the effective integration of participants from a diverse array of disciplinary perspectives; (2) an integrated approach to defining problems as a system of interrelated structures, functions, and processes; (3) the collaborative learning required to synthesize a comprehensive understanding of the interrelated components and processes of the system containing the problem(s); (4) a process of co-writing that guides the alteration of socio-ecological systems to eliminate the problem(s); and (5) communication that clearly and persuasively conveys the message required to actualize resolution.
\end{abstract}

Keywords Creativity $\cdot$ Communication $\cdot$ Interdisciplinary collaboration $\cdot$ Knowledge application $\cdot$ Problem solving $\cdot$ Recursive writing process $\cdot$ Systems thinking

\section{Introduction}

We shape the future through creative thought; by conceiving the reality we desire. In a time of global uncertainty, visionary concepts of a more sustainable future become an indispensable aspect of contemporary thought. Conceptions shape reality in two ways: by establishing expectations about what might be, and by guiding actions to achieve it. The role of the design disciplines is to determine the shape of a future that improves some aspect of the present. Designers formulate their ideas by visualizing a future reality. These visions-scenarios- describe not just what that reality might be but also what improvements it might bring about and how it might be realized. Creative scenarios, like knowledge, are among our most powerful tools for improving the relationships between people and the environment;

Don R. Day IBM (retired).

Journal of Socio-Ecological Practice Research (SEPR) Special Issue: Our alternative futures in the 2020s and beyond: scenarios of socio-ecological practice in an uncertain world.

Michael D. Murphy

MurphyLandArch@gmail.com

1 Department of Landscape Architecture and Urban Planning, Texas A\&M University, College Station, Texas, USA

2 IBM, Mason, Texas, USA relationships that are deteriorating more rapidly than is generally recognized. This paper examines how the process of design can help shape a brighter future.

Despite a century of exponential growth in knowledge, with scientific publications doubling over the last decade (Bornmann and Rüdiger 2015), the socio-ecological problems confronting society have not only grown more extensive, they have also become more intractable (IPCC 2014). But science, our window into the workings of the world, often operates as if nature was organized like universities. Disciplines, however, do not represent different aspects of reality but different ways of viewing it; nature exists as a complex, interactive whole (Ackoff 1999, p. 33). As a consequence, neither the abundance nor the availability of knowledge has led to resolution of these increasingly threatening problems. To address these challenges will require not just new knowledge but new ideas, as expressed through the creative contributions of deeply insightful people to integrate the vast but disparate knowledge available and guide formulation of the creative scenarios required to inform sustainable change and improvement (Kanter 2006).

A conventional view of reality that conceives nature as comprehensible in disciplinary categories has led to some of our most intractable problems. For example: over half a billion people go to bed hungry each night (FOA 2020). Improved agricultural production and distribution seems 
the clear path to their survival. Unfortunately, agricultural production as it is currently practiced—of which roughly $30 \%$ is wasted prior to consumption (Van den Bos Verma et al. 2020)—along with forestry, accounts on a global basis, for our greatest consumption of water and a quarter of greenhouse gas emissions (IPCC 2014). Conventional, energy-intensive, non-regenerative methods of food and fiber production are also significant contributors to the loss of biodiversity that reduces the stability and resilience on which ecosystems, and thus societies depend (Butchart et al. 2010; Hooper et al. 2012). There is much to trouble us about this situation. At its root is the inertia of our conventional approach to reality and science-and to survival-that is faltering under the strain of a dynamic and rapidly deteriorating global environment.

Exceptional circumstances require extraordinary measures. For future scenarios to drive successful policy and practice for socio-ecological management, profound changes are needed in the way these scenarios are conceived and expressed. Beyond just mending the broken relationship between people and the environment, these scenarios will require radical, creative insight about the comprehensive reformulation of life on Earth. Conventional "solutions" are not "solving" contemporary problems. In fact, they perpetuate them (Dick et al. 2016). Moreover, because science and the media are motivated by divergent agendas, they find themselves estranged, leading to disparities in one another's mutual understanding. As a consequence, science is poorly reported and thus, poorly understood among the wider audience of the public at a time when it is most essential (Hartz and Chappell 1997; Peters 2013).

Entrenched cultural behavior represents one of the greatest obstacles to seeing problems in a new way, and thus to their creative resolution (Kinzig et al. 2013). Approaching a situation in an innovative way, however, requires a shift in social norms. It also requires experimentation and risk taking. It is creative thinking - the driver of all progress-that is critical to the formulation of scenarios that make knowledge systemically useful (Hennessey and Amabile 2010). If we are to believe Einstein, "Imagination is more important than knowledge." Unfortunately, imaginative speculation runs counter to conventional behavior within the scientific and practice communities. Because creative thinking violates prevailing cultural norms, speculation poses significant risks to both credibility and careers (Vraga et al. 2018). Any creative act deviates from the status quo. In the culture of scholarship, the status quo obliges researchers to retain their objectivity through detachment and silence. This reticence removes those with the greatest scientific insight from the policy and practice conversation, creating a knowledge gap in the science-practice relationship (Bertuol-Garcia et al. 2018).
Bringing researchers and practitioners together in a joint effort could help bridge this gap. However, practice careers are built on predictable performance and creative thinking produces unanticipated outcomes (Rothenberg et al. 1996, p. 23; Andreasen 2005 p. 17). The risk for practitioners is that the process of scenario formulation becomes less predictable. To bring researchers, who are not just unfamiliar but uncomfortable with speculation about the future, and practitioners, whose careers are built on predictable outcomes, into the uncertainties of a creative scenario-writing process requires careful attention to their integration if they are to be adequately sheltered from criticism from within their disciplinary domains; which is necessary if they are to have the freedom to think and speak openly and innovatively. This, however, is not an easy task when the practitioner's or researcher's identity, credibility, and rewards derive primarily from the limited perspective of their disciplines, not from their participation on or the outcomes of a particular initiative.

Subliminal social pressure-normative social influence-encourages people to conform their views to those prevailing in their social or disciplinary milieu (Aronson et al. 2005). Conformity is an unconscious adaptive response motivated by an instinct for self-protection (Griskevicius et al. 2006), one of the most powerful motivators of human behavior (Maslow 1987; Agostinelli et al. 1992; Alicke and Sedikides 2009). Because it contributes to group cohesion by limiting conflict within the social setting and protecting the individual from isolation, conformity is the norm (Heuser 2005). But an emphasis on self-protection and group cohesion comes at the cost of objectivity and independent thinking, and thus, to the creative thinking so essential to breaking free of convention (Rothenberg 2015).

And just as researchers must learn how to interact productively with practitioners, practitioners must learn how to take full advantage of the contributions of researchers. Neither the time and energy required to create such a collaboration, nor its outcomes can be predicted accurately, and as a consequence, are more difficult to integrate into the constraints of even conventional practice performance. But only a fresh approach that breaks the norms of conformity and convention is likely to lead to better outcomes. It is when scenarists are free to engage in experimentation and risk-taking that they will discover a new path to the future.

To respond effectively to the pressing need, a successful scenario must be visionary enough (i.e., effective as well as novel), and comprehensible enough (i.e., communicated through accessible, compelling language) to convey its message broadly and gain the support necessary for its acceptance and execution. To achieve the bold, innovative approach that conditions demand, scientists must be able to speculate lucidly from their area of expertise, liberated from the limitations of conventional expectation and the 
often-inaccessible language of science, just as practitioners must be freed from the artificial constraints of time or the bureaucratic language of practice.

This paper outlines the features of such a path-a scenario-writing process-with the potential to facilitate the innovation required to create ideas that shape a more successful integration of culture and environment. It identifies some of the obstacles to be overcome and describes measures needed to free the creative spirit necessary to envision a sustainable future. To formulate the creative scenarios that successfully resolve the looming crises threatening society will require decisive action, comprehensively and aggressively communicated. For scenarios to drive successful policy and practice in the management of socio-ecological systems, their formulation will depend as much on the approach taken to understand these problems as on the actions taken to resolve them. One of the primary considerations is to begin with a creative mindset.

We look first at what constitutes creative thinking.

\section{Creativity}

Stephen Jay Gould reminds us that, "Science is not a heartless pursuit of objective information. It is a creative human activity, its geniuses acting more as artists than as information processors" (Gould 1977, p. 201). On a fundamental level, creativity can be defined by the laws of thermodynamics as a negentropic process, resulting in an increase in order and complexity. Ian McHarg describes creativity as the generative interaction of energy and matter, the result of syntropic action: the tendency towards energy concentration, order, and organization; a creatively fitting condition he describes as a "least work, maximum success solution" (McHarg 1996, p. 244).

Creativity is nominally defined as a phenomenon comprising at least four aspects (Rothenberg and Hausman 1996; Mumford 2003, p. 110; Andreasen 2005, p.17; Kaufman et al. 2006; Hélie and Sun 2010):

- It produces something original; it is different, new, innovative

- It is useful in bringing about improvement in an existing situation

- It is valued for the improvement it brings about

- It results in a product of some kind, either tangible or intangible.

The product-invention, artwork, theory-is necessary to a determination that a creative act has occurred, but on an individual basis, creativity is an attribute not of products but of mental processes (Nanay 2013; Kaufman 2013). Creativity is also described as an action: "Creativity is any act, idea, or product that changes an existing domain, or that transforms an existing domain into a new one." (Csikszentmihalyi 1996, p. 28). The human capacity for creativity is the cognitive ability to conceive and realize an original idea to resolve a problem or capitalize on an opportunity. It is that capacity that to a significant extent, accounts for the extraordinary success of our species.

Creativity is expressed in two general forms: generative creativity whereby new ideas are originated, and elaborative creativity whereby a novel idea is developed and applied (Ellamil et al. 2011; Sowden et al. 2015). Both forms play a critical role in research - the creativity of discovery (Rothenberg 2015)_ just as they are necessary for effective scenario formulation.

\subsection{Characteristics of creative thinking}

Creative thinking is characterized by a cluster of mutually reinforcing cognitive capacities (Torrance 1965; 1970):

- Fluency: the ability to produce a number of different ideas relevant to specified requirements within a limited period of time.

- Flexibility: the ability to produce ideas in a variety of categories, shifting easily from one mind set or conceptual response to another; to use cognitive processes, knowledge, or materials in diverse ways.

- Originality: the ability to produce novel, statistically infrequent responses; unusual, unique ideas that are unconventional: the unanticipated ideas that "no one else would think of."

- Elaboration: the ability to formulate a variety of different ways to examine, expand, and develop a concept in the detail necessary to fully establish its utility and appropriateness to application.

- Resistance to closure: the ability to keep an open mind and get past the first idea that comes to mind and exhaust all conceivable options in the search for even greater possibilities.

These different cognitive capacities are thought to operate integratively as a comprehensive system. In a collaborative effort, each of these capacities is enhanced by the interaction of people with different areas of expertise and problemsolving approaches. As will be shown in Sect. 4, below, each of these attributes is critical to the development of a creative scenario.

\subsection{Creative thinking process}

Nearly a century ago, Graham Wallas described the mental processes employed by creative people across a broad range of fields to produce novel ideas or resolve complex 
problems. He identified four areas of cognitive control as key stages in the creation of new ideas (Wallas 1926, p. 80):

- Preparation: accumulating relevant information and preparing the mind by focused attention on the salient features of a problem.

- Incubation: internalizing the relevant information and allowing the mind to work through the problem by subconscious awareness.

- Illumination: discovering new relationships, often described as occurring as a flash of inspiration, as new patterns are recognized.

- Verification: determining whether a new idea is likely to be successful, and through refinement and elaboration, bringing it to a form suitable for application.

With this model the initial preparation stage is characterized by a period of intense activity to identify, assemble, organize, and evaluate information, preferably from multiple points of view. If, at this stage, a solution is found, the problem is solved. However, if the problem is too complex or ill-defined and the problem solver reaches an impasse, incubation begins with progression toward the next stages of the process (Hélie and Sun 2010).

The four stages of thinking operate as an integrated set: a system of creative thinking that occurs at both conscious and subconscious levels. Creative thought involves the application of different cognitive modes through the interplay of conscious and non-conscious processes (Baumeister et al. 2007), both important to a successful outcome. Nonconscious priming makes consciously creative thought more accessible. This is significant in the application of a rulebased problem-solving process (as described below) because mastering the rules of a protocol can be helpful in breaking free of them: a precondition of creative thought and innovation. The conscious appreciation of a rule-based approach can also be helpful in ensuring a novel response (Baumeister et al. 2007). The interaction of these different types of thinking is one of the key features of creative thinking process.

It is significant that the nonconscious cognitive system operates in parallel with conscious thinking although the two modes function differently. The nonconscious can work on unrelated tasks simultaneously (Lieberman et al. 2002), unlike the conscious system, which operates serially. "Apparently human creativity flourishes because of the interplay between two systems - one automatic and nonconscious, the other conscious and controlled, and both indispensable" (Baumeister et al. 2007, p. 150). If applied to scenario writing, a consciously creative approach has the potential to enhance both the thinking process and its outcomes. What this means to the researcher is that in creative exploration, intuition is to be trusted rather than shunned. But creative expression can only exist in an environment in which there is freedom to examine what might appear to be outlandish ideas in the search for a better one.

Creative thinking is dependent on an attitude of openness to experience, which is "absolutely essential to creativity" (Kaufman and Gregoire 2015, p. 83). It is the single greatest predictor of creative achievement (Batey and Furnham 2006; Kaufman 2013; Kaufman et al. 2015). But openness to experience requires the assurance of safety to engage in it without undue risk. Two conditions essential to constructive creativity are psychological safety and freedom of expression (Andreasen 2005, p. 128). To foster creative outcomes, a successful scenario-writing process creates and sustains these conditions.

Next, we examine how researchers, practitioners, and policy makers might interact in a process of creative scenario writing.

\section{Creativity in problem solving}

The complexity of nature is too great to be understood without an analysis of its disciplinary components and interactions. Although the rigors of objectivity and disciplinary analysis are critical to the creation of knowledge, it is equally essential that once available, knowledge is applied holistically if it is to address the complexity of reality. It is imperative that the scientific community broadens its restrictive way of thinking and approaching problems, not just in its disciplinary perspective, but also in how it conceives its role in the application of knowledge. Researchers will need to be more intimately involved in knowledge application if change is to bridge the knowledge-practice gap to address the complexity of interactions between culture and environment, and overcome the accelerating deterioration of global ecosystems, exacerbated by social conflicts on a scale and severity never before experienced (Watson 2005).

Recent examples of researchers collaborating to broaden the base of consideration may be seen in the work of the international climate research community in their development of a scenario framework to facilitate integrated research and consistent assessment to inform policy. Over the past decade, the group began with brief narratives to formulate projections for a few key variables (GDP, population, urbanization). After assessing the framework's effectiveness, researchers expanded their investigation to add new indicators (governance, income distribution, access to basic services, air pollution) when it became clear that the contributions of additional disciplines were required. This scenario framework has been successful in shaping climate change research (O'Neill et al. 2020). Nevertheless, the limitations of domain specificity and how it constrains interdisciplinary research remain impediments to collaboration due 
to ingrained institutional and cognitive barriers (MacLeod 2018).

\subsection{Interdisciplinary collaboration}

Problems are not disciplinary. Because the environment exists as a complex whole, to achieve sustainability in one area, such as ecological well-being, requires that commensurate attention is given to others, such as social and economic conditions (Gupta and Vegelin 2016). In the management of a complex system, such as a landscape, or an urban setting, comprehensive understanding of their diverse system components and interrelationships requires expertise outside the practitioner's disciplinary domain, just as it is beyond the grasp of an individual discipline. To resolve this deficiency assumes a collaborative approach through which the problem is addressed by orchestrating the diverse areas of knowledge required for understanding the disciplinary parts of the problem as an integrated set of features, processes, and relationships (Garb et al. 2008).

What is required is a vast intuitive leap among multiple segments of society: researchers, practitioners, policy makers, and just as importantly, within the population at large (Kanter 2006). When reality is portrayed as categoricalsuch as energy problems or pollution problems-there is widespread although mistaken belief that these interrelated problems can be understood and resolved independently. And because socio-ecological change is based on the extent of its acceptance, scenarios also need to include meaningful participation by the people to be affected (Ndubisi 1997, p. 38). In a rapidly changing world where the conventions of disciplinary and political division and isolation have become one of our greatest obstacles to progress, the challenge for human creativity is to determine how best to engage our ever-expanding knowledge to sustainably integrate culture and nature. It is through collaboration that the assumption of one another's roles is promoted, that in turn, stimulates innovation and the flow of new ideas (Etzkowitz 2008). Interdisciplinarity has become widely recognized as an important approach to fostering creativity in team performance and innovation (Oddane 2015; Clapp and Jimenez 2016; Runco 2017; Edmondson and Harvey 2018).

Beyond the explanation of facts, the most important test of theory is its ability to predict (Worrall 1989; Douglas 2009). The theory needed to inform future scenarios must improve prediction, and it must do so systemically. Only when knowledge is holistically integrated is it useful in predicting the behavior of complex human and natural systems. There are, however, some fundamental problems to be overcome in applying knowledge from different disciplines successfully (Capra 1982; Murphy 2016, p. 220). These include:

- The diversity of disciplines and knowledge areas.
- Profoundly different approaches to the creation of knowledge.

- Absence of an institutional framework for knowledge.

- Lack of understanding about the values encoded in information.

- Lack of understanding about what is known and what is unknown.

- Inability to predict what information is most urgently required.

To successfully apply knowledge to formulate an effective scenario will require the best minds as well as the most contemporary knowledge at our disposal (Csikszentmihalyi 1996, p. 102). And who better to expedite the process of integration and understand the nuance of theory and how it might be applied than those who have created and validated it? Just as importantly, who is better positioned to anticipate the refinements to theory that are required continually in response to the dynamics of a rapidly changing reality and the knowledge to describe it? What is required is the creative ability "to extract order from chaos..." (Kaufman et al 2015, p. xxiii), which is only possible from a position of deep understanding. Perhaps the greatest advantage of researchers' participation in scenario writing lies in their ability to ask the incisive questions prompted by their disciplinary perspectives. The deeper one's understanding, the quicker they are to see connections about where the problems or opportunities lie. On the other hand, "most breakthroughs are based on linking information that is not thought of as related" (Csikszentmihalyi 1996, p. 329).

\subsubsection{The outsider advantage}

Expertise does not eliminate bias (Dror 2020). The capacity for rapid and efficient performance due to the automaticity of the expert can also introduce tunnel vision that restricts flexibility, causing them to miss or ignore important information (Kapur et al. 2011). Due to factors such as expert bias: the tendency to see things in a traditional way, a high percentage of innovative discoveries are made by outsiders (Lewandowsky and Kirsner 2000; Sassenberg and Moskowitz 2005; Bilalić et al. 2008; Dane 2010; Hennessey and Amabile 2010; Acar and van den Ende 2016). When collaborations comprise participants from diverse areas of expertise, they are all outsiders. With the creators of theory from different disciplines directly involved with practitioners and policy makers in a collaborative scenario-writing process, the likelihood of discovering unlikely connections and shaping a sustainable future outcome is substantially improved.

But as a practical consideration, outsiders must be brought together as a unified problem-solving organization if they are to effectively address a common problem. The basic features of problem-solving that serve as integrating 
influences to focus the interactions of collaborators include (Broadbent 1973; Smith and Brown 1993):

- Recognizing and correctly defining problems and their underlying causes

- Determining the conditions to be satisfied to eliminate the problem

- Devising a strategy/course of action-a scenario-to achieve the desired conditions

- Communicating the strategy to satisfy the conditions required for resolution

At each stage of the problem-solving process, the direct contributions of the scientific community are essential for comprehensive, long-term success. It is insufficient for researchers to create knowledge and then leave it to others to insightfully interpret and apply. Moreover, because social and ecological systems are inherently dynamic as well as complex, they require the deep understanding resident in a broad array of disciplinary domains to either understand or to resolve systemically (Musacchio et al. 2005, p. 329; Calkins 2012, p. 19). To integrate the depth of understanding needed to define problems comprehensively and formulate a successful resolution strategy, planning and design practice have demonstrated that the most effective approach is to incorporate those possessing the necessary expertise directly into the problem-solving process (Zeisel 1988, p. 32; Marrs, et al. 1989).

Since each discipline views the environment and its role in understanding or changing it in a different way-what one sees in the environment lies as much within the mind as in the environment (Meinig 1976) — each one sees a different reality, and as a consequence, only part of reality; each blind man perceives a different elephant. Because knowledge has been created in disciplinary categories, it will require the collaboration of those who have created that knowledge to reconstitute it into an integrated form more closely representing the reality of experience. Similarly, it will require the expertise of practitioners and policy makers to translate resolution concepts into reality.

In the broad scheme of things, each discipline has its unique areas of strength and knowledge, and as a consequence, its own weaknesses and limitations. To accentuate their strengths and make up for weaknesses, interdisciplinary teams are formed to broaden the base of expertise and resolve problems in a more comprehensive way (Van der Ryn et al. 1996) - the only way that socio-ecological problems can be solved.

\subsubsection{The advantage of diversity}

Collaborative organizations are systems that capitalize on mutual learning to minimize the difficulty associated with managing complexity and uncertainty (Wheatley 1992). The intent of a collaborative systems approach is to determine the complex interrelationships of the environment to be changed and to manage them as an integrated set to yield the desired outcomes (Ackoff 1981; Gharajedaghi 2005). Collaborators operating with different thinking modes and expertise in different knowledge areas have the potential to significantly enrich the process of creative problem solving, introducing a number of important benefits into the scenario-writing process. Foremost among these include (Senge 1990; Edmondson and Harvey 2018):

- Broaden the spectrum of expertise and perspectives being brought to bear on understanding the problem

- Deepen the level of understanding and insight when those most knowledgeable are directly involved in the process

- Increase the rigor and breadth of critical evaluation when those with different areas of expertise approach the problem from diverse perspectives

- Expand the thinking strategies and modes available to recognize innovative pathways to understanding and resolution

- Increase the potential for continuous learning as participants from different disciplines interact in a dialogue leading to a holistic understanding of the problematic situation.

Dialogue inevitably translates the process of scenario formulation into a shared learning experience; the participants become a learning organization (Senge 1990). This is critical to a scenario's success because the information on which it is based continuously expands in a working environment of dynamic interaction and exchange. The interaction among diverse disciplinary perspectives improves the likelihood of a more integrated understanding when competing worldviews are synthesized into a unified vision of the problem. Collaboration advances knowledge because more learning takes place among multiple disciplines working together than when individuals or disciplines work alone (Senge 1990, p. 14; Parker 1994, p. 23).

Learning organizations generate integrated knowledge drawn from individual contributors as their shared vision merges into something new: synergistically synthesized knowledge (Hedfors and Granvik 2008, p. 23). Interdisciplinary collaboration is a knowledge-building as well as a knowledge-based process of problem solving. And, importantly, through dialogue, lapses in knowledge are identified that prompt additional research to improve understanding and decision-making. Moreover, creating scenarios serves as an innovative form of scholarly enquiry to identify novel lines of research (Ramirez et al. 2015; O'Neill et al. 2020). Creative thought in science is "not a mechanical collection 
of facts and induction theories, but a complex process involving intuition, bias, and insight from other fields" (Gould 1977, p. 125). Viewed in this way, concerns about professional ostracism might be less constraining. The rigors of scientific method can influence practice and policy profoundly given the appropriate opportunity.

\subsubsection{Difficulties with collaboration}

The benefits of collaboration notwithstanding, interdisciplinary effort is fraught with difficulties, not the least of which is the tendency to rely on successful past experience, which tends to be formed in disciplinary isolation. Added to this are the issues of disciplinary competition and distrust, entrenched disciplinary values, and perhaps the most difficult to predict, idiosyncratic egos. Effective collaboration requires a strategy for reducing the reluctance to forego "proven" disciplinary practices and accept an innovative approach to scenario formulation, the results of which cannot be predicted. Experience with these problems has led to the development of some tactics for reducing resistance to innovation. These include the use of a collaborative process that emphasizes (Bright 1988):

- Perceived advantage: how collaboration provides benefits that a disciplinary approach cannot

- Compatibility: how team collaboration fits with and builds on existing disciplinary methods or models

- Simplicity: the process must be easy to comprehend as a concept, expanding rather than substituting prevailing conventions

- Divisibility: the collaboration process should be applicable one step at a time, building on itself in phases

- Communicability: the process should be described using conventional vocabulary rather than disciplinary jargon to make it easier for all to understand and accept

- Relative Cost: the process should cost no more than conventional methods with failure to accept the process seen as a higher cost than acceptance

- Consequence of Failure: the consequence of failure should not present an unacceptable risk to the credibility or humiliation of participants

Incorporating these working procedures can enhance collaboration. Furthermore, these considerations are equally important to the success of communication between collaborators and their intended audience(s) in disseminating the results of their work since the same resistance factors apply to the acceptance of a new way of managing socioecological conditions.

Knowledge is created to improve decisions as well as understanding, both of which are improved through dialogue. The new knowledge advances the creativity of discovery, but also through the practical application of knowledge, expands and enhances its influence. However, there remain risks to researchers who participate directly in the formulation of scenarios for which no direct evidence yet exists. And there is the consideration of the uncertainty of knowledge. This kind of participation removes the researcher from the security of evidence-based demonstration that a hypothesis about some future reality is a reliable assumption on which to speculate, even though the evidence available may suggest it. But the dialogue of collaboration presents a personal risk for both researchers and practitioners: it takes place in the presence of others. As Linus Pauling advised, the trick is to have lots of ideas and then to know which ones to throw away (Crick 1995). Interdisciplinary collaboration denies the researcher the safety of seclusion from which to speculate and evaluate concepts before determining which ideas to reveal and which ones to discard. Alternatively, collaborators benefit from the insights of others in making a determination about which ideas have the greatest promise. When diverse perspectives are synthesized into a comprehensive view it creates the opportunity to develop a unified understanding of the problematic situation. But there is a caveat to a collaboration being successful in developing this synthesis of knowledge.

One of the critical factors to successful collaboration is the selection of the participants. A major challenge for interdisciplinary collaboration is the need to reconcile and integrate the individual members' strongly held disciplinary views. Some of the factors that determine a collaboration's effectiveness include: its organizational culture, the nature of the shared work, the clarity of their undertaking, group autonomy, and performance feedback (Sundstrom et al. 1990). But the most important indicator of an effective team environment is the quality of interactions among the participants (Forsyth 1990). The strong group dynamic required for effective interaction is dependent on a working relationship of mutual trust and acceptance (Bertcher and Maple 1996). Although a collaboration is most creative when it operates with a few clear principles and a great deal of individual freedom (Wheatley 1992), the greatest likelihood that it will realize its full potential is when all the participants share a strong personal commitment to their common enterprise (Katzenbach and Smith 1993).

To engage a collaborative process in design problem solving, it is helpful to recognize that disciplines have cultures analogous to biological DNA-cultures that each discipline will continually seek to reproduce unless their cultural DNAs themselves are altered (Ackoff 1999, p. 17; Gharajedaghi 1999, p. 16). In addition to its value in providing a comprehensive understanding of a problem, a systems approach provides a strategy for altering cultural DNA in a way that reconstitutes the collaborators' view of their common problem. 
For collaborators to create a systemic understanding of the problem requires a systems approach.

\subsection{Systems thinking in collaborative problem solving}

Scientific analysis focuses on taking things apart to study and understand them. Systems thinking focuses on understanding how the parts behave and interact: It provides a framework for approaching problems as systemic wholes (Forrester 1988). Systems thinking focuses on investigating those aspects of a system required for holistic understanding of the structure, function, process, and context of the problematic situation, and then managing them as an integrated set (Gharajedaghi 2005). A systems approach seeks not to solve problems directly but to redesign the system containing the problematic situation, "so as to eliminate the problem. Such a design incorporates common sense and scientific research, and increases our learning more than trial-and-error or scientific research alone can do" (Ackoff 1999, p. 22).

Russell Ackoff demonstrated that successful innovation comes from transforming the problematic system as a whole, not from tinkering with its parts. "Systems" replaces "analysis" as the method for developing innovations in the operational improvement of complex organizational structures (Ackoff 1974). This approach to the management of complex systems may be described as reconciliation design: the holistic redeployment of a system's features and processes to optimize their relationships under altered conditions (Rosenzweig 2003; Grimm et al. 2008). The whole system is best understood by viewing it from all perspectives simultaneously (Ackoff 1974), which interdisciplinary collaboration facilitates in considerable depth.

Because we cannot see the future, we create it by design (Gabor 1963, p. 207). West Churchman (1971) showed that one of the most effective ways to learn about a system is to design it. The aim of understanding and re-designing a system is to create conditions that "persuade" the system to move away from a state of malfunctioning order by transitioning to a desired state in which the problem no longer exists. When systems thinking dissolves the boundaries between disciplinary points of view, it creates a pathway to the holistic understanding required for resolution. When creative thinking is conducted as a participative process with representatives from different disciplines approaching the problem from their individual domain perspectives, there needs to be a practical approach to orchestrating their interactions if they are to become integrated into a unified problem-solving/scenario-writing process. This can be facilitated by their active engagement in three critical actions:
- Problem definition: assure that participants from all the relevant disciplines are involved in defining the nature of the problem, creating a shared vision and thereby, making each of them stakeholders in the outcome

- Problem solution: solicit suggestions about how to resolve the problem from participants from all disciplinary positions

- Solution evaluation: incorporate multiple evaluation and feedback cycles into the deliberation process, through which the participant's different disciplinary insights are given repeated opportunity for expression.

These activities facilitate the dialogue needed to synthesize the collaborator's mutual learning about the holistic nature of the problem, and about their different ideas for a solution, both of which are required for a systemic resolution. Scenario formulation needs to function as a reiterative process of interdisciplinary speculation, evaluation, and feedback that when successful, creates a transdisciplinary understanding of the problem.

Developing design resolution concepts through repeated speculation and feedback cycles, in what Ackoff describes as a successive approximation process, has the benefit of continually incorporating new information and fresh insight - each iteration effectively revealing the situation in hindsight — before additional iterations are undertaken to formulate new, better informed scenarios.

The operational dilemma becomes a question of how to impose sufficient discipline to assure efficient use of the time available for research, speculation, communication, and evaluation while simultaneously providing adequate opportunities for mutual learning, reflection, and resolution: essentially, how to organize the design problem-solving/scenariowriting process to enable collaborators to be simultaneously integrated, mutually informed, efficient, and creative.

Now we examine what that process might look like.

\section{A recursive scenario writing process}

Accomplished writers such as E. B. White and Ernest Hemingway, have advised that the best writing is rewriting. Hemingway considered it the only kind of writing. This recursive scenario-writing process follows that admonition. The design process outline described here is proffered as a model for the orchestrated thinking approach needed to integrate researchers, practitioners, and policy makers into an effective learning/scenario-writing organization. Since solving a complex problem inevitably requires multiple attempts to arrive at a final determination, the process is typically - and usually most successfully-executed as an iterative process.

The design problem-solving process doesn't manage creativity, but it manages the creation of opportunities for 
the interactive dialogue needed to prompt the discovery and evaluation of the novel ideas required to understand and resolve what have been aptly described as "wicked problems" (Rittel and Webber 1973). Wicked problems, which are dynamic, difficult to understand and thus to define, present situations that have only better or worse solutions-with the better ones being established on the basis of their potential to be both implemented and sustained as well as on their likelihood to resolve. Even the better solutions, however, lead to additional challenges. Furthermore, a solution that works today is unlikely to work in the future when conditions have evolved (Buchanan 1992). In particular, the process enables the comparison of possible future courses of action, as evaluated by objective, multidimensional criteria to reach a decision-formulate a scenario- that has a reasonable probability of success. Each cycle of a successive approximation process provides renewed opportunities to view the problem from the participants' multiple perspectives, as well as providing the group with multiple opportunities for interaction, inspiration, feedback, and learning.

But even under the best of circumstances, collaboration among parties with diverse interests, personalities, and disciplinary perspectives is a challenging prospect. To reduce the level of difficulty and assure some measure of success requires a practical strategy for integrating collaborators into the unified mindset necessary to develop a transdisciplinary understanding of the system to be changed and facilitate the creative thinking necessary to formulate a scenario to effect that change. Such an approach requires a process that:

\section{- Enables the formation of an effective collaboration} among the participants

- Guides a collective process of defining the problem areas and their systemically linked relationships

- Facilitates the shared learning required to develop a synthesized understanding of the related components of the system and how they interact

- Establishes a process for determining how the system might be altered to bring about resolution.

Under conventional practice conditions, design decisionmaking is guided by a sequence of related tasks (Marrs et al. 1989; Smith and Browne 1993; Partidario and Clark 2000; Seip and Wenstop 2007). The design process suggested here is organized so that each task prepares for the one that follows, leading designers through a logical progression of learning, exploration, and ultimately, decision making; first about the nature of the problem, then about the conditions needed to solve it, and finally, about the form that resolution might take.

Design (scenario) effectiveness can only be definitively established after an intervention has been realized and in operation. In architecture, this determination process is referred to as post-occupation evaluation (Preiser et al. 2015). In the case of socio-ecological practice, this takes the form of longitudinal system monitoring to determine if an intervention is yielding the intended outcomes (Menard 2008). Monitoring provides the basis for continued adjustments to the system to accommodate the dynamics of the environment. But the final stages of the design process outlined below-the final concept selection, implementation, and performance evaluation stages of the design problem-solving sequence-are not a part of this discussion. The emphasis here focuses on the first four stages: actions to develop a continuous sequence of feedback loops that provide the learning opportunities on which the creative breakthrough necessary for a successful scenario can be discovered.

The outline below describes a typical sequence of design problem-solving tasks involved in formulating plausible alternative futures. The process begins by taking the problem apart to determine its features and processes, then, through design speculation, synthesizes the parts into a new form to determine their integrated meaning. In practice, the different thinking tasks tend to be continuous with each one seamlessly transitioning into the next. But for descriptive purposes, the tasks are defined individually as stages of design thinking: landmarks in the progression of the process. The basic stages of a design process that make provision for the learning and reflection required to bring the issues into focus include (Murphy 2016, p. 197):

Stage 1 Recognize the problem: the process is initiated with an initial charge to designers, normally expressed as a formal statement that a condition requiring intervention exists (recognition). This stage requires awareness of the problem.

Stage 2 Define the problem components: investigate the problematic situation to identify and assess the salient issues, uncover critical relationships, and describe the conditions/performance requirements necessary for successful resolution (preparation). This stage involves critical thinking and analysis.

Stage 3 Search for alternative solutions: envision possible innovations to satisfy the problem requirements and develop representations to describe their conceptual possibilities to satisfy the intended performance outcomes (speculation). This stage requires generative creativity: fluency, flexibility, originality, resistance to closure.

Stage 4 Evaluate alternative design options: identify the proposed concepts' strengths and weaknesses to accelerate reflection and deliberation. If no satisfactory solution is found, reformulate the solution or the problem definition based on insights revealed by evaluation (incubation). This stage requires critical thinking and analysis. 
Stage 5 Select the preferred alternative: based on evaluation, determine the concept that best resolves the problem as defined (illumination). This stage requires agreement among collaborators that it satisfies their individual and collective criteria for success.

Stage 6 Develop the selected design concept: refine or elaborate the innovation within the constraints of its particular context to ensure its applicability (verification). At this stage the specific conditions of implementation-available technology, political will, costs, timeare examined and integrated into the resolution concept. This stage requires elaborative creativity.

Stage 7 Implement the solution: alter the system (realization). The implementation stage requires communication and actions on multiple fronts over time to effect system change.

Stage 8 Evaluate the outcomes: measure the system outcomes over time (evaluation). This stage requires critical assessment of environmental performance in response to the changes imposed.

The first four stages of the design-process model serve as a framework for scenario writing. It incorporates both critical and creative thinking modes, as conducted through the four types of thinking (in the Wallas model) needed for creative resolution. When the process is repeated, each iteration creates additional opportunities for engagement and dialogue among practitioners, researchers, policy makers, and communicators at each stage of the scenario development process. There are two indispensable goals of the cyclical design process:

- Provide confirmation that the problem has been accurately identified and systemically described

- Provide an effective solution-a scenario-with the potential to resolve the problem

When conducted iteratively, the first four stages of the process (Fig. 1) provide multiple opportunities to develop alternative scenarios, each of which can be evaluated, not just by the performance requirements established in Stage 2 (Problem Definition), but also by comparison to one another to expand the basis on which a scenario is finally determined.

A recursive scenario writing process organizes the search for a solution through which speculation about a possible resolution is posited, evaluated, and ultimately, either accepted, or if not, leads to another attempt at resolution; or the problem may be reformulated for further investigation until a successful concept has been found (Gharajedaghi 2005). The process seeks not only to formulate a scenario to inform system change, but also to continually refine the collaborators' understanding of the

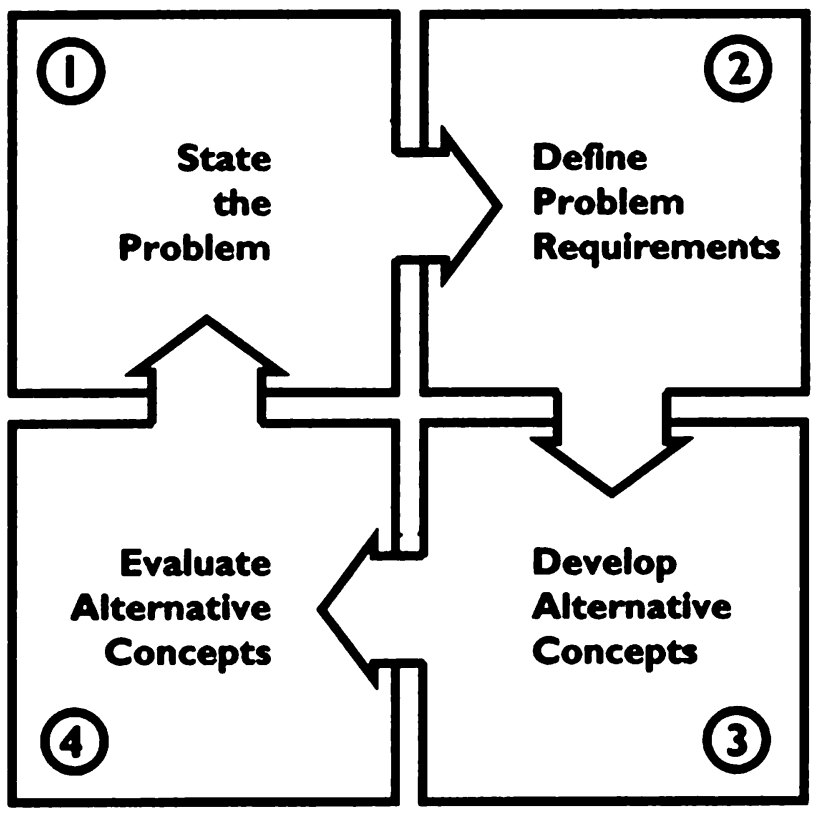

Fig. 1 Cyclical repetition of the four-stage ideation phase of scenario development (Murphy 2016, p. 211)

problem to be resolved; one of the primary benefits of a continuous learning process is to pose the most insightful questions. Only when the appropriate questions are asked do the most effective answers becomes apparent.

By formulating a series of possible scenarios-effectively, straw men to be critically examined-collaborators create the "evidence" on which to reflect at each cycle of the process. And, significantly, when formed by collaboration, each scenario represents an increasingly holistic concept; not just the selective examination of a particular aspect of the problem. This approach leads researchers to create scenarios that are intentionally provocative where the goal is to stimulate discussion about strategies and risk mitigation. Through reflection and evaluation, collaborators essentially "mine" concepts for additional information and insight, first as the basis for further investigation to correctly define the problem, and ultimately, in their search for its solution. Moreover, multiple cycles create multiple opportunities for serendipitous discoveries that have not been consciously sought, but by happenstance, sometimes emerge from wide-ranging examination of the problem from different disciplinary perspectives (Rothenberg 2015). Significantly, such fortuitous discoveries are only likely if the observers are well prepared to recognize an opportunity when it presents itself: "It should be clear that an individual without the requisite elements in his [or her] response repertoire will not be able to combine them so as to arrive at a creative solution" (Mednick 1962, p. 222). As Louis Pasteur (1854) so famously remarked, “... chance favors only the prepared mind." And the greater 
the range and depth of observers' expertise, the greater chance is favored.

Although the source of a creative idea cannot be predicted, or in many cases even identified when collaboratively derived, what the process does is to ensure that the scenario ultimately selected will have enhanced probabilities for being seen as the most effective approach from multiple vantage points (Fig. 2).

Knowledge is redefined through the synthesis of research, prior experience, and creative speculation. More importantly, it imbues degrees of relevance by assaying its potential for application. The stages of design thinking function, not so much as a sequence but as multiple points of access to view the problem from multiple-increasingly informedperspectives for further investigation and innovation.

The four ideation stages are cycled repeatedly until consensus is reached and an acceptable resolution concept has been mutually agreed, or until no new information or ideas are discovered. Learning and improvement are possible at each stage with each cycle of the process. It is also fundamentally important that the transition to Stage 5 (Select the preferred alternative) is deferred until all possibilities have been exhausted. Premature commitment effectively blocks objective evaluation of alternative scenarios, as well as the acceptance of any new understanding of the problem if it deviates from that on which a preferred concept has been based. Resistance to closure is one of the key characteristics of creative thinking (Torrance 1970). As a consequence of the mutual learning that takes place, it is not uncommon that

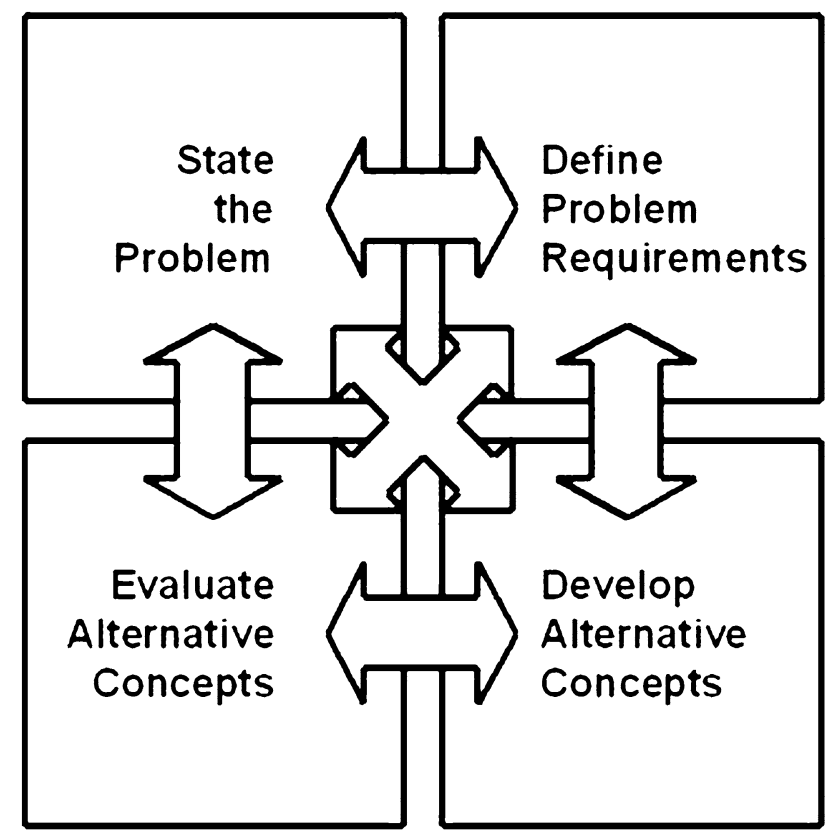

Fig. 2 Multiple nonlinear options for interaction between design thinking modes (Murphy and Hedfors 2011, p. 10) a final determination is reached by general consensus as a logical outcome of the first four stages of the process, rather than as a specific activity designated for that purpose.

Once a satisfactory concept has been agreed (Stage 5), the process moves into the detailed refinement stage to formulate and communicate the scenario. It must also be recognized that design thinking - scenario writing — will continue into the implementation stage as monitoring feedback reveals the need for modifications in response to unforeseen discoveries and the unpredictable nature of the environment in which change is being made.

Finally, we examine how a scenario might be communicated.

\section{Communication}

Communication is key to scenario success. For example, although the potential for a greenhouse gas effect has been understood for over a century (Arrhenius1896), with evidence of global warming becoming established by the 1980s and widely available by the 21st Century (McKibben 1989; Emanuel 2007; Fagan 2008; Hansen 2009), there has been little action to reduce the negative impacts of a warming climate at any useful scale. As "the Captain" (actor, Strother Martin) says in an old movie, "What we have here is a failure to communicate" (Cool Hand Luke 1967).

Because different members of the receiving audience for a communication may be expected to have differences in background for understanding information, as well as differences in their world view and values, the message may or may not resonate with a particular segment of the wider audience. If we consider the deep cultural, political, and economic divisions that exist, any number of issues can arise to interfere with successful communication. For these and many other reasons, particular attention must be paid, not just to the message but also to the way the message is communicated.

Communication as a process is a dynamic interaction influenced by multiple variables. According to a traditional model (Shannon and Weaver 1949), the process is initiated when a communicator sends a message intended to inform a receiver of information, which has been encoded for the purpose of transmission. The sender selects a medium or channel for conveying the message considered most likely to be effective in transmitting the information accurately and completely. The receiver of the message decodes the information being conveyed and provides feedback to confirm receipt and understanding, and hopefully, that action will follow; or to request additional information or clarification. The heart of Shannon's theory is a simple model of communication: The transmitter encodes information into a signal, which is corrupted by noise and then decoded by the receiver. His model incorporates two key insights: isolating 
the information and noise sources from the communication system to be designed, and modeling both of these sources probabilistically (Tse 2020).

According to the Shannon-Weaver model, the major elements of communication process are: (1) sender, (2) information, (3) encoding, (4) communication channel, (5) receiver, (6) decoding, and (7) feedback. An error at any step of the process can interfere with communication success. If the message of the scenario is unsuccessfully conveyed to its intended audience(s) its purpose is put at risk. With multiple audiences, such as national, state, and local officials as well as representatives of commerce, industry, and individual participants involved in the execution of actions informed by a scenario, the opportunities for error are compounded. The sender must bear in mind that all receivers will interpret the message differently, based on their cultural experience, and individual world view (Wurman 1990, p. 247).

The message here is that a scenario-writing process that includes participation by researchers and practitioners with expertise in communication, particularly via popular and social media has an improved chance of success. Communication must be understood as an integral part of the problem and as a consequence, it must be an integral part of a solution.

There are two areas of communication critical to scenario writing: (1) internal communication to facilitate interactions within the scenario-writing group, and (2) external communication to the various members of the audience for whom the scenario is intended. Both require attention to receiving as well as sending information.

\subsection{Internal communication}

Interdisciplinarity requires that each member of the team takes responsibility for identifying the relevant issues as revealed by their disciplinary perspective and explaining them effectively to one another. Because contributions from all collaborators are necessary to the creation of a comprehensive view of the problem, it is essential for participants to remain mindful that their particular area of expertise is unlikely to be well understood by other collaborators and to ensure that the necessary information is conveyed clearly across disciplinary lines. This requires the use of conventional vocabulary whenever possible to avoid disciplinary jargon that might be misunderstood (Bright 1988).

Effective interaction requires the use of a common language equally understandable to collaborators as well as to the larger external audience. As disciplines become more sophisticated and distinct, they develop unique terms for communicating complex concepts as a form of shorthand. This means that team members uninitiated in a particular discipline may be unable to interpret the jargon, particularly if terms have different meanings to different groups.
To become effectively integrated into a team collaboration, participants must fully understand the positions of one another. This requires more than merely allowing other members to present their views; it also requires that participants assume responsibility for listening proactively, with an open mind, in order to understand fully the insights of others. Unless collaborators achieve this level of mutual understanding, it will limit their ability to integrate the implications of another's insights into their own understanding.

One of the most important aspects of successful interaction is positive, two-way flow of information among the participants. However, care must be exercised in their method of exchange since poorly structured interaction is not only time consuming and unproductive, it interferes with understanding and actually discourages further communication. The challenge lies in how to encourage more communication rather than more discussion. Alternatively, discussion that is overly abbreviated in the interest of saving time limits understanding.

Resolving environmental problems involves addressing interrelated ecological and social systems operating on multiple scales. Progress on these problems can be made only through a concerted effort to change personal and social norms. One of the pressing demands of communication is to engender a shift in social norms in order that new ideas can become accepted and behavior changed (Kinzig et al. 2013), a consideration that applies to both internal and external communication.

\subsection{External communication}

Canadian communication theorist Marshall McLuhan popularized the aphorism, "the medium is the message," by which he meant that any communication medium "amplifies or accelerates existing processes," introducing a "change of scale or pace or shape or pattern into human association, affairs, and action," thereby effecting change (McLuhan 1964, pp.8-9).

From clay tablets to crayons to TV to browsers of the World Wide Web, efforts in the communication of ideas seem to follow the principle that the selected medium itself has a large influence on how the message engages (or fails to engage) an intended audience. However playful McLuhan's premise may seem, it is nevertheless essential to consider how to effectively communicate-if not market- the results of research that urges the necessary attention of those who can act on it. If the medium fails to reach, the message fails to land.

One pitfall in planning the communication strategy for science writing is to presume that communication has occurred solely by the act of publishing results in a journal or slide deck. The problem is that publishing does not equate to persuasion, and citation does not equate to influence. A 
study in the role of citations as indicators of research quality and impact concluded that "an increased use of citation indicators in research evaluation and funding may imply less attention to these other research quality dimensions, such as solidity/plausibility, originality, and societal value" (Aksnes et al. 2019).

Traditional scholarly publishing through journals, while essential for access by other researchers, fails to provide visibility to the public, and often sequesters the information within paywalls, inhibiting popularization in the broader public debate.

"If a paper is cited, this does not imply it has actually been read. According to one estimate, only 20 per cent of papers cited have actually been read. We estimate that an average paper in a peer-reviewed journal is read completely by no more than 10 people. Hence, impacts of most peer-reviewed publications even within the scientific community are minuscule" (Biswas and Kirschherr 2015).

Nevertheless, science results do find a steady means of promotion to the public. The potential sphere of influence for environmental scenarios includes not only other researchers but also media and the general public.

\subsubsection{Options for external communication}

Scientific writing covers a wide gamut of both internal scientific and public communication, which itself spans from mediated science journalism to direct public outreach. (Peters 2013).

Scientific journals remain the primary means for publishing research, but the model has evolved to accrue a reputation: subscription costs for journals have increased dramatically, causing the medium to be viewed "less like dissemination than restriction" (Trench 2008, p.4). The model challenges researchers to consider more expansive approaches to publishing. For example, the Scholarly Publishing and Academic Resources Coalition (SPARC) aims to "maximize the number of scientists, scholars and students who have access to our articles ... [the journal] will be extraordinarily liberal in its dissemination rules" (Rosenzweig 1999, p.1).

Cultural evolution research offers some insights on bettering the spread of scientifically accurate information to help effect cultural change. The science of science communication studies evidence-based practices as a means to convey scientific findings and methods that inspire responsive behavior. Considering that educational and cultural biases tend to be a popular barrier to forming thoughtful policy for managing global warming activities, this research might suggest more broadly useful strategies for writers of environmental scenarios. Focus areas include content properties of the particular information or behavior, the attitudes and worldviews at play, and the social norms and incentives that influence culturally appropriate responses to, say, a climate change scenario that applies particularly to that audience. "What makes a communication effort successful on one science-based issue, say vaccination, is not necessarily relevant for another issue, say climate change-although some content biases (e.g., eliciting of emotions) may be relevant for several different issues. This challenge forces science communicators to be knowledgeable about the unique "root causes" of the specific issues about which they are communicating..." (Bendixen 2020, p.3).

Writing to influence in the public forum implies creating a degree of tension between the traditional and the contemporary models of communication. In this regard, knowing and trusting your audience can promote the message. "Whereas a traditional model of science communication supposed that uncertainty was progressively reduced, even eliminated, in the movement from relatively closed to relatively open spheres of communication, a contemporary model acknowledges both that much of the science of most interest to the public is inherently uncertain, and that science-interested publics - and perhaps even the citizenry in general-are capable and willing to handle such uncertainty." (Trench 2008, p. 12).

The publication tools that have evolved in the milieu of the content-democratizing influence of the internet now support not only scientific communication in its privileged communities but also various means of public science communication, through a variety of strategies.

Science journalism retreats a bit from the direct aspect of communication by invoking mediation-a reporter, an interlocutor - to negotiate the message of the researcher to the interests of the listener or the person best able to implement scenarios. Science journalism represents an amalgam of public interest, educational policy, marketing, and even lobbying that provides popular outreach. Today's science journalist is a capable partner: "It is now common for science journalists to come to the field after completing advanced degrees in science." (Hayden and Hayden 2018, p. 2).

Science organizations and individual scientists address the public directly online through creation of Web sites and blogs, participation in social networks, and uploading of videos that compete with the content provided by professional science journalism (Peters 2013).

\subsubsection{To inform or to influence}

A debate that divides technical communication from marketing communication is whether the goal of writing is to inform (how to) or to influence (create a commitment to an idea or investment). Scientific communication benefits from the same self-examination with respect to use of scenarios. 
The argument to influence: In a sense, directly engaging the public with scientific research is a form of Content Marketing, a term that hews closely with McLuhan's observation that knowledgeable use of the medium itself influences the effectiveness of the message. Marketers do not just inform, they influence with content that leads to a call for action, whether it is a sale or a commitment to align with a venture, such as environmental cares. As seen from a tutorial on writing for marketing, influence is about leveraging the power of a person or brand to move possible outcomes into more favorable adoption. In marketing, this influence is about the Call to Action (downloading a white paper, viewing a video); in scenario writing, influence is about making more desirable outcomes attractive (or contrariwise, representing dire outcomes as something to be avoided) (iResearch Services 2019).

The Aristotelian principles of logos (logic/evidence), ethos (credibility/goodwill), and pathos (emotion) still apply for creating effective, persuasive writing. Stated practically, this means (Hurley 2020):

- Argue your case with strong evidence and clear logic

- Present yourself as credible, competent, and cooperative

\section{- Generate positive emotions in your readers}

"Persuading people isn't about getting people to take an action that they don't want to do, without any justifiable reason. Persuasion does mean laying out a clear, logical case for why someone should do something, explaining the facts and letting people draw their own conclusions" (Jones 2016, p.1).

The argument to inform: What is the goal of communicating scientific discoveries beyond the scientific community (or to practitioners influence by science)? Research that develops understanding of a subject can certainly inform policy, but to what extent should it attempt to influence policy?

Again, building out from McLuhan's proposition about how the medium influences the message, the choice of what to study, if it is of sufficient interest to a reading public, can engage that public to action. In other words, finding the right thing to talk about can result in both informing and influencing policy. (Rice 2018).

\section{Conclusion}

Formulating scenarios provides a systematic way of envisioning and examining alternative futures. By envisioning them, we bring them closer to reality. If we fail to plan for a more successful future, and fail to make full use of the knowledge we possess, by default, we plan to fail. There is a pressing need to exploit knowledge holistically if the enormously complex problems we face are to be successfully addressed. Scenarios provide an important tool both for clarifying plans and for bringing them to a wider audience.

Science is dedicated to discovering the laws of nature. Mathematics searches for new theorems to describe them. The practical disciplines such as architecture, engineering, and planning, apply these laws to resolve human problems. The different disciplines are distinct and yet interdependent. Claude Shannon (Shannon and Weaver 1949) was the rare individual who contributed to all three: science, mathematics, and engineering, and in doing so, laid the foundational infrastructure of the information age (Tse 2020). Although it may be unrealistic to expect that most people would able to contribute significantly in even one of these areas, any level of contribution can be of value. There need to be greater opportunities within the disciplines for crossover and collaboration - at least by those who are inclined-if their different disciplinary contributions are to be successfully infused into a coherent and holistic approach to recognizing and solving the wicked socio-ecological problems we face.

Ultimately, an interdisciplinary, systems approach to scenario writing will succeed only if the participants are fully committed to their shared enterprise and prepared to subsume their individual egos or disciplinary allegiancesbut not their disciplinary expertise-in the service of their joint effort. The dissolution of conventional disciplinary perspectives to create a holistic approach to problem solving requires significant effort on the part of all the participants. When successful, an interdisciplinary approach to systemically understand and creatively resolve wickedly complex problems leads to recognition that, in a dynamic world, problems do not remain "solved." Consequently, the approach described here should be understood, not as a path to the resolution of socio-ecological problems but to their continuous examination and management. The repair of adverse relationships between culture and environment is not an event but an evolutionary process of adaptation based on learning and feedback to improve fitness with a changing environment. And as with natural evolution, some innovations will not prove successful. The strength of the global ecosystem will depend primarily on the maintenance of complexity and interrelatedness for its ability to respond to needed future corrections in the process of sustaining harmony in a dynamic environment.

Finally, there is the consideration of detached objectivity in science. Participation by the researcher does not equate to advocacy when collaborating to create a more comprehensive understanding of the problems society faces or in speculating about a remedy to address them; particularly when carried out under the active scrutiny of a well-informed investigative body. For science to be a cornerstone of policy and practice decisions, it must maintain 
the credibility of accurate, unbiased information as paramount to its effectiveness. But unless researchers find a path to more effectively infuse knowledge into policy and behavior, the quality and accuracy of science will be irrelevant to action-as we have observed with regard to the COVID-19 pandemic. The unprecedented extent and peril of the current situation imposes new responsibilities and priorities on the socio-ecological practice community. When science is fully and continuously engaged in both the creation and the application of knowledge, policy and practice will be in a stronger position to employ the rapidly increasing knowledge at our disposal to imagine a new way forward, giving the world what Daniel Pink describes as "something it didn't know it was missing" (Pink 2018). Design thinking can amplify and facilitate that process.

\section{References}

Acar OA, van den Ende J (2016) Knowledge distance, cognitive-search processes, and creativity: The making of winning solutions in science contests. Psychol Sci 27(5):692-699

Ackoff RL (1974) Redesigning the future. Wiley, New York

Ackoff RL (1981) Creating the corporate future. Wiley, New York

Ackoff RL (1999) In Nicholson M, Myers K (eds.) Proceedings of the Russell L Ackoff and the advent of systems thinking conference: A celebration of the work of Russell L Ackoff and developments in systems theory and practice. The College of Commerce and Finance, Villanova University, Villanova, Pennsylvania

Agostinelli G, Sherman SJ, Presson CC, Chain L (1992) Self-protection and self-enhancement biases in estimates of population prevalence. Pers Soc Psychol Bull 18:631-642

Aksnes DW, Langfeldt L, Wouters P (2019) Citations, citation indicators, and research quality: An overview of basic concepts and theories. SAGE Open 9(1):2158244019829575

Alicke MD, Sedikides C (2009) Self-enhancement and self-protection: What they are and what they do. Eur Rev Soc Psychol 20:1-4

Andreasen NC (2005) The creative brain: The science of genius. Plume, Washington, DC

Aronson E, Wilson TD, Akert AM (2005) Social psychology, 5th edn. Prentice Hall, Upper Saddle River, New Jersey

Arrhenius S (1896) On the influence of carbonic acid in the air upon temperature of the ground. Philosophical Mag J Sci 5(41):237-276

Batey M, Furnham A (2006) Creativity, intelligence, and personality: A critical review of the scattered literature. Genet Soc Gen Psychol Monogr 132(4):355-429

Baumeister RF, Schmeichel BJ, De Wall CN (2007) Is the conscious self: a help, a hindrance or an irrelevance to the creative process? Adv Psychol Res 53:137-152

Bendixen T (2020) How cultural evolution can inform the science of science communication-and vice versa. Humanities Soc Sci Commun 7(135):1-10

Bertcher HJ, Maple FF (1996) Creating groups, 2nd edn. Sage Publications, Thousand Oaks, California

Bertuol-Garcia D, Morsello C, El-Hani CN, Pardini R (2018) A conceptual framework for understanding the perspectives on the causes of science-practice gap in ecology and conservation. Biological revue 93(2):1032-1055
Bilalić M, McLeod P, Gobet F (2008) Inflexibility of experts-Reality or myth? Quantifying the Einstellung effect in chess masters. Cogn Psychol 56:73-102

Biswas A, Kirchherr J (2015) Prof, no one is reading you. The Straits Times. https://www.straitstimes.com/opinion/prof-no-one-isreading-you

Bornmann L, Rüdiger M (2015) Growth rates of modern science: A bibliometric analysis based on the number of publications and cited references. J Am Soc Inf Sci. https://doi.org/10.1002/ asi.23329

Bright JR (1988) Tactics of innovation: How to introduce new ideas to people who don't want them. Industrial Management Center, North Edgecomb, Maine

Broadbent G (1973) Design in architecture. Wiley, London

Buchanan R (1992) Wicked problems in design thinking. Des Issues $8(2): 5-21$

Butchart SHM et al (2010) Global biodiversity: Indicators of recent declines. Science 328(5982):1164-1168

Calkins M (2012) The sustainable sites handbook: A complete guide to the principles, strategies, and best practices for sustainable landscapes. Wiley, Hoboken, New Jersey

Capra F (1982) The turning point: Science, society and the rising culture. Simon and Schuster, New York

Churchman CW (1971) Design of inquiring systems. Basic Books, New York

Clapp EP, Jimenez RL (2016) Implementing STEAM in makercentered learning. Psychol Aesthet Creat Arts 10(4):481-491

Cool Hand Luke (1967) Warner Brothers-Seven Arts movie directed by Stuart Rosenberg, produced by Gordon Carrol with screenplay by Donn Pearce and Frank Pierson

Crick F (1995) The impact of Linus Pauling on molecular biology. Presentation to the Salk Institute. Special collections, Oregon State University Libraries, Corvallis, Oregon

Csikszentmihalyi M (1996) Creativity: Flow and the psychology of discovery and invention. Harper Collins, New York

Dane E (2010) Reconsidering the trade-off between expertise and flexibility: A cognitive entrenchment perspective. Acad Manag Rev 35:579-603

Dick M, Rous AM, Nguyen VM, Cooke SJ (2016) Necessary but challenging: Multiple disciplinary approaches to solving conservation problems. FACETS 1:67-82. https://doi.org/10.1139/ facets-2015-0003

Douglas HE (2009) Reintroducing prediction to explanation. Philos Sci 76(4):444-463

Dror IE (2020) Cognitive and human factors in expert decision making: Six fallacies and the eight sources of bias. Anal Chem 92(12):7998-8004

Edmondson AC, Harvey J (2018) Cross-boundary teaming for innovation: Integrating research on teams and knowledge in organizations. Hum Resour Manag Rev 28(4):347-360

Ellamil MC, Dobson C, Beeman M, Christoff K (2011) Evaluative and generative modes of though during creative process. NeuroImage. 5 August 2011. www. Elsevier.com/locate/ynimg

Emanuel K (2007) What we know about climate change. MIT Press, Cambridge, Massachusetts

Etzkowitz H (2008) Triple helix: University-industry-government innovation in action. Routledge, New York

Fagan B (2008) The great warming: Climate change and the rise and fall of civilizations. Bloomsbury Press, New York

Forrester JW (1988) Designing Social and Managerial Systems, System Dynamics Group, Sloan School. MIT, Cambridge MA

Forsyth D (1990) Group dynamics, 2nd edn. Brooks/Cole, Pacific Grove, California

(FOA) Food and Agriculture Organization of the United Nations, IFAD, UNICEF, WFP and WHO (2020) Food security and 
nutrition in the world. Rome: FAO, IFAD, UNICEF, WFP and WHO. ISBN: 978-92-5-132910-8

Gabor D (1963) Inventing the future. pp .207, Alfred A Knopf, New York

Garb Y, Pulver S, Van Deveer SD (2008) Scenarios in society, society in scenarios: Toward a social scientific analysis of storylinedriven environmental modeling. Environ Res Lett 3(4):1-8

Gharajedaghi J (1999) in Nicholson M, Myers K (eds.) Proceedings of the Russell L Ackoff and the advent of systems thinking conference: A celebration of the work of Russell L Ackoff and developments in systems theory and practice. The College of Commerce and Finance, Villanova University, Villanova, Pennsylvania

Gharajedaghi J (2005) Systems thinking: Managing chaos and complexity, a platform for designing business architecture. Butterworth-Heinemann/Elsevier, Burlington, Massachusetts

Gould SJ (1977) Ever since Darwin: Reflections in natural history. WW Norton, New York

Grimm NB, Faeth SH, Golubiewski NE, Redman CL, Wu JG, Bai X, Briggs JM (2008) Global change and the ecology of cities. Science 319:756-760

Griskevicius V, Goldstein NJ, Mortensen CR, Cialdini RB, Kendrick DT (2006) Going along versus going alone: Whenever fundamental motives facilitate strategic (non) conformity. J Personal Personal Psychol 91:281-294

Gupta J, Vegelin C (2016) Sustainable development goals and inclusive development. Int Environ Agreem: Politics, Law Econ $16: 433-448$

Hansen J (2009) Storms of my grandchildren. Bloomsbury, New York

Hartz J, Chappell R (1997) Worlds apart: How the distance between science and journalism threatens America's future. First amendment center, Nashville, Tennessee

Hayden T, Hayden E (2018) Science journalism's unlikely golden age. Front Commun 2:24. https://doi.org/10.3389/fcomm.2017.00024

Hedfors P, Granvik M (2008) Landscape architecture theory: A source for interdisciplinary sustainable design, in Fabris L, Enviroscape: A manifesto, 2nd blu + verde International Congress. Politecnico di Milano, Faculty of Architecture, Department BEST, Milan, Italy

Hélie S, Sun R (2010) Incubation, insight, and creative problem solving: A unified theory and a connectionists model. Psychol Rev 117(3):994-1024

Hennessey BA, Amabile TM (2010) Creativity. Annu Rev Psychol 61:569-598

Heuser BL (2005) Social cohesion and voluntary associations. Peabody J Educ 80:16-29

Hooper DU, Adair EC, Cardinale BJ, Byrnes JEK, Hungate BA, Matulich KL, Gonzales A, Duffy JE, Gamfeldt L, O'Connor MI (2012) A global synthesis reveals biodiversity loss as a major driver of ecosystem change. Nature 486(7401):105-108

Hurley P (2020), The most effective writing relies on these 3 persuasive techniques. https://www.hurleywrite.com/Blog/346103/Themost-effective-writing-relies-on-these-3-persuasive-techniques

(IPCC) Intergovernmental panel on climate change (2014) AR5 Climate change 2014: Impacts, adaptation, and vulnerability

iResearch services (2019) The art of influencing \& not informing. (September 27) https://www.iresearchservices.com/the-art-ofinfluencing-not-informing

Jones M (2016) How to be persuasive, and why every type of professional needs this skill. https://climb.pcc.edu/blog/how-to-bepersuasive-and-why-every-type-of-professional-needs-this-skill

Kanter RM (2006) Innovation: the classic traps. Harv Bus Rev 84(11):72-83

Kapur N, Manly T, Cole J, Pascual-Leone A (2011) The paradoxical brain-so what? In, Kapur, N (ed) The paradoxical brain. (Medicine) Cambridge: (pp 177-188). Cambridge University Press: Cambridge UK
Katzenbach JR, Smith DS (1993) The discipline of teams. Harvard Bus Rev. Mar-Apr 1993

Kaufman SB (2013) Opening up openness to experience: A fourfactor model and relations to creative achievement in the arts and sciences. J Creative Behavior 47(4):233-255

Kaufman SB, Gregoire C (2015) Wired to create: Unveiling the mysteries of the creative mind. Perigee, New York

Kaufman JC, Sternberg RJ (2006) The international handbook of creativity. Cambridge University Press, New York

Kaufman SB, Quilty LC, Grazioplene RG, Hirsh JB, Gray JR, Peterson JB, DeYoung CG (2015) Openness to experience and intellect differentially predict creative achievement in the arts and sciences. J Pers. https://doi.org/10.1111/jopy.12156

Kinzig AP, Ehrlich PR, Alston LJ, Arrow K, Barrett S, Buchman TG, Daily GC, Levin B, Oppenheimer M (2013) Social norms and global environmental challenges: The complex interaction of behaviors, values, and policy. Bioscience 63(3):164-175

Lewandowsky S, Kirsner K (2000) Knowledge partitioning: Contextdependent use of expertise. Mem Cognit 28:295-305

Lieberman MD, Gaunt R, Gilbert DT, Trope Y (2002) Reflection and reflexion: A social cognitive neuroscience approach to attributional inference. Adv Exp Soc Psychol 34:199-249

MacLeod M (2018) What makes interdisciplinarity difficult? Some consequences of domain specificity in interdisciplinary practice. Synthese 195:697-720

Marrs CB, Mulder CE, Murphy MD (1989) Conservation planning: Integrating human and land resources in South Africa's developing areas. In Proceedings of the international federation of landscape architects

Maslow AH (1987) Motivation and personality. Harper \& Row, New York

McHarg IL (1996) A Quest for life: An autobiography. Wiley, New York

McKibben B (1989) The end of nature. Anchor Random House, New York

McLuhan M (1964) Understanding media: The extensions of man. Signet Books, New York

Mednick SA (1962) The associative basis of creative process. Psychol Rev 69:220-227

Meinig DW (1976) The beholding eye: Ten versions of the same scene. Landscape Archit 66:47-54

Menard S (ed) (2008) Handbook of longitudinal research: Design, measurement, and analysis. Elsevier, Burlington, Massachusetts

Mumford MD (2003) Where have we been, where are we going? Taking stock in creativity research. Creat Res J 15(2-3):107-120

Murphy MD (2016) Landscape architecture theory: An ecological approach. Island Press, Washington, DC

Murphy MD, Hedfors P (2011) Systems theory in landscape architecture. Conference: Urban nature. Los Angeles: Council of educators in landscape architecture

Musacchio L, Ozdenerol E, Bryant M, Evans T (2005) Changing landscapes, changing disciplines: seeking to understand interdisciplinarity in landscape ecology change research. Landsc Urban Plan 73:326-338

Nanay B (2013) Between perception and action. Oxford University Press, New York

Ndubisi F (1997) Landscape ecological planning. In: Thompson GF, Steiner FR (eds) Ecological design and planning. Wiley, New York

O’Neill BC, Carter TR, Ebi K, Harrison PA, Kemp-Benedict E, Kok K, Kriegler E, Preston BL, Riahi K, Sillmann J, van Ruijven BJ, van Vuuren D, Carlisle D, Conde C, Fuglestvedt J, Green C, Hasegawa T, Leininger J, Monteith S, Pichs-Madruga R (2020) Achievements and needs for the climate change scenario framework. Nat Clim Chang 10:1074-1084 
Oddane TAW (2015) The collective creativity of academics and practitioners in innovation projects. Int J Manag Proj Bus 8(1):33-57

Parker GM (1994) Cross functional teams: Working with allies, enemies, and other strangers. Jossey-Bass, San Francisco

Partidario MR, Clark R (2000) Perspectives on strategic environmental assessment. CRC Press, New York

Pasteur L (1854) Inaugural lecture as professor and dean of the Faculty of Science, Univ of Lille, France. 7 Dec 1854. Perterson NH (ed) 1963. A treasury of the world's great speeches, p. 473. Grolier, New York

Peters HP (2013) Gap between science and media revisited: Scientists as public communicators. Proceed National Acade Sci 110(3):14102-14109

Pink DH (2018) When: The scientific secrets of perfect timing. Riverhead Books Penguin Random House, New York

Preiser WF, White E, Rabinowitz H (2015) Post-occupancy evaluation. Routledge, London

Ramirez R, Mukherjee M, Vezzoli S, Kramer AM (2015) Scenarios as a scholarly methodology to produce "interesting research." Futures 71:70-87

Rice K (2018) Research should inform, not influence. ...and then there's physics (blog), https://andthentheresphysics.wordpress.com/ 2018/08/05/research-should-inform-not-influence/

Rittel HWJ, Webber MM (1973) Dilemmas in a general theory of planning. Policy Sci 4(2):155-169

Rosenzweig ML (1999) Editorial, evolutionary ecology research. 1: 1. https://www.evolutionary-ecology.com/ddeditor.pdf

Rosenzweig ML (2003) Win-win ecology: How the Earth's species can survive in the midst of human enterprise. Oxford University Press, New York

Rothenberg A, Hausman CR (eds) (1996) The creativity question. Duke University Press, Durham, North Carolina

Rothenberg A (2015) Flight from wonder: An investigation of scientific creativity. Oxford University Press, New York

Runco MA (2017) Comments on where the creativity research has been and where is it going. J Creative Behavior 51(4):308-313

Sassenberg K, Moskowitz GB (2005) Don't stereotype, think different! Overcoming automatic stereotype activation by mindset priming. J Exp Soc Psychol 41:506-514

Seip KL, Wenstop F (2007) A primer on environmental decision making: An integrative quantitative approach. Springer Press, New York

Senge PM (1990) The fifth discipline: The art and practice of the learning organization. Doubleday, New York

Shannon CE, Weaver W (1949) The mathematical theory of communication. University of Illinois Press, Champaign, Illinois

Smith GF, Browne GJ (1993) Conceptual foundations of design problem solving. IEEE Trans Syst Man Cybern 23(5):1209-1219

Sowden PT, Pringle A, Gabora L (2015) The shifting sands of creative thinking: Connections to dual process theory. Think Reason 21:40-60

Sundstrom E, De Meuse KP, Futrell D (1990) Work teams: Applications and effectiveness. Am Psychol 45(2):120-133

Torrance EP (1965) Scientific views of creativity and factors affecting its growth. Daedalus 94(3):663-681

Torrance EP (1970) Creative learning and teaching. Harper Collins, New York

Trench B (2008) Internet: turning science communication inside-out? In: Bucchi M, Trench B (eds.) Handbook of public communication of science and technology. Routledge, London \& NY. ISBN 978-0-415-38617-3 http://doras.dcu.ie/14807/

Tse D (2020) How Claude Shannon invented the future: Today's information age is only possible thanks to the groundbreaking work of a lone genius. Quanta, December 22

Van den Bos VM, de Vreede L, Achterbosch T, Rutten MM (2020) Consumers discard a lot more food than widely believed: Estimates of global food waste using an energy gap approach and affluence elasticity of food waste. PLoS ONE. https://doi.org/ 10.1371/journal.pone.0228369

Van der Ryn S, Cowan S (1996) Ecological design. Island Press, Washington, DC

Vraga EK, Myers TA, Kotcher J, Beall L, Maibach EW (2018) Scientific risk communication about controversial issues influences public perceptions of scientists' political orientations and credibility. Royal society open science 5(2): 170505. Published online 2018 Feb 21. doi: https://doi.org/10.1098/rsos.170505

Wallas G (1926) The art of thought. Harcourt, Brace, New York

Watson RT (2005) Turning science into policy: challenges and experiences from the science-policy interface. Philos Trans 360(1454):471-477

Wheatley MJ (1992) Leadership and the new science: Learning about organization from an orderly universe. Berrett-Koehler, San Francisco

Worrall J (1989) Fresnel, Poisson, and the white spot: The role of successful predictions in the acceptance of scientific theories: The uses of experiment. Cambridge University Press: 135-157

Wurman RS (1990) Information anxiety: What to do when information doesn't tell you what you need to know. Bantam Books, New York

Zeisel J (1988) Inquiry by design: Tools for environment-behavior research. Cambridge University Press, New York

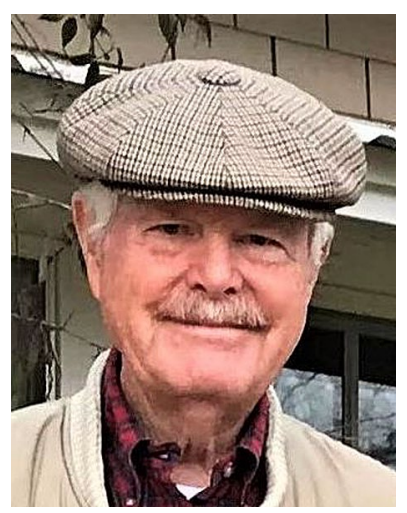

Michael Murphy is professor emeritus of the Department of Landscape Architecture and Urban Planning at Texas A\&M University. His academic and research interests include design theory, ecological planning and design, collaborative problem definition, and design decision process. During the four decades of his active career, he served as head of the Department of Landscape Architecture at the University of Pretoria in South Africa and the Department of Landscape Architecture and Urban Planning at Texas A\&M University in the USA. His most recent book is Landscape Architecture Theory: An Ecological Approach, published by Island Press.

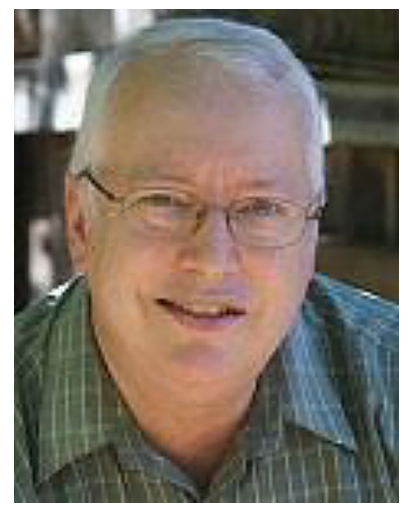

Don Day Ranking among the top XML technology leaders today, Don Day not only contributes to the success of individual projects and products, his influence can also be found on nearly every XML initiative in production. His efforts have resulted in multiple patents, official recognition for his contribution to reengineering IBM's information assets, and designation as an OASIS Distinguished Contributor for convening and leading the OASIS Darwin Information Typing Architecture (DITA) Technical Committee. Don is well known for advising and consulting on strategy, technology, and best practices for optimizing the value and usefulness of unstructured data. Don consults on content design and engineering and is a regular public speaker and thought leader. 